\title{
Erratum
}

\section{RX J0042.3+4115: a stellar mass black hole binary identified in M 31, and other papers}

\author{
R. Barnard ${ }^{1}$, U. C. Kolb ${ }^{1}$, and J. P. Osborne ${ }^{2}$
}

\begin{abstract}
1 The Department of Physics and Astronomy, The Open University, Walton Hall, Milton Keynes, MK7 6BT, UK
2 The Department of Physics and Astronomy, The University of Leicester, Leicester, LE1 7RH, UK
\end{abstract}

In this work we have previously identified low mass X-ray binaries (LMXBs) in XMM-Newton observations of M 31 from their characteristic power density spectra (PDS). These PDS were characterised by a broken power law, with the spectral index changing from $\sim 0$ to $\sim 1$ at a break frequency in the range $0.01-1 \mathrm{~Hz}$, and signify LMXBs in their low accretion-rate state (van der Klis 1994 , ApJ, 92, 511).

However, the observed PDS were false positives resulting from the improper treatment of non-simultaneous lightcurves; the corrected PDS are featureless (see Barnard et al. 2007, A\&A, 469, 875, for details). As a result, there is no evidence for black hole primaries in the subjects of these papers.

Key words. X-rays: general - X-rays: binaries - accretion, accretion disks - galaxies: formation - black hole physics errata, addenda 